\title{
Effect of the CYP3A5, CYP3A4, CYP3A7, ABCB1, POR and NR1/2 genes in the phar- macokinetics of tacrolimus in a pediatric cohort with stable serum concentrations after renal transplantation: study protocol.
}

\author{
Irene Dapia ${ }^{1,+}$, Aleksandra Tabakov ${ }^{2,+}$, Laura Espinosa Román ${ }^{3}$, Lucía Díaz ${ }^{4}$, Pedro Arias Lajara ${ }^{1}$, Marta Melgosa ${ }^{3,}$ An- \\ gel Melgar ${ }^{3}$, Carlota Fernández ${ }^{3}$, Alicia Herranz Estellés ${ }^{5}$, Pablo Lapunzina ${ }^{1}$, Alberto M. Borobia ${ }^{4,6 \ddagger}$, Antonio J. Car- \\ $\operatorname{cas}^{4,6 \ddagger}$ \\ ${ }^{1}$ Medical and Molecular Genetics Institute (INGEMM). La Paz University Hospital. Madrid. \\ ${ }^{2}$ Clinical Pharmacology Department. Gomez Ulla Hospital. Madrid \\ ${ }^{3}$ Pediatric Department, La Paz University Hospital, IdiPAZ. \\ ${ }^{4}$ Clinical Pharmacology Department. La Paz University Hospital. School of Medicine. Universidad Autónoma de Madrid. IdiPAZ \\ ${ }^{5}$ Clinical Trial Unit. Clinical Pharmacology Department. IdiPAZ \\ ${ }^{6} \mathrm{SCReN}$ (Spanish Clinical Research Network) \\ ${ }^{\dagger}$ Contributed equally to this work \\ ${ }^{\ddagger}$ Corresponding authors: Antonio J. Carcas Sansuan, MD, PhD - Alberto M. Borobia, MD, PhD, Clinical Pharmacology Department. La Paz University \\ Hospital of Madrid, Paseo de la Castellana, 261.28046-Madrid emails: antonio.carcas@uam.es; alberto.borobia@salud.madrid.org
}

\section{ABSTRACT}

BACKGROUND: Therapeutic response to pharmacological therapy in humans shows large intrapatient and interpatient variability both in treatment efficacy and adverse drug reactions (ADR). Part of this variability can be explained by genetic polymorphisms in genes encoding TAC metabolism related proteins. The aim of this study is to evaluate the contribution of genetic variation in the CYP3A4, CYP3A5, CYP3A7, POR, NR1/2 and $A B C B 1$ genes to this variability in order to achieve a better understanding of TAC pharmacokinetics and a more personalized approach for TAC dosing in a cohort of pediatric patients with stable serum concentrations after renal transplantation.

METHODS AND DESIGN: This is a unicenter retrospective cross-sectional study. The protocol was approved by the Clinical Research Ethics Committee of the La Paz University Hospital (Madrid, Spain) and will be carried in this same hospital. 50 pediatric patients with stable serum concentrations after renal transplantation are expected to be included. Peripheral blood samples will be collected for molecular analysis (pharmacogenetics studies) and AUC estimation (C0, C1 and C3 hours after TAC administration).

DISCUSSION: To date there are not dosing algorithms that can explain accurately TAC metabolism. The incorporation of a complete pharmacogenetic (PhGx) profile into these algorithms may help in the individualization and optimization of TAC treatment in pediatric renal transplant patients.

STUDY REGISTRATION: FC/HULP_002/2014

Keywords: Tacrolimus, transplantation, pharmacokinetics, pharmacogenetics, cytochrome P450, metabolism. Received: December, 22 2016; Accepted: January, 10 2017; Published: January, 162017.

Copyright: (c) 2017 Authors. This is an open-access article distributed under the terms of the Creative Commons Attribution License, which permits unrestricted use, distribution, and reproduction in any medium, provided the original author and source are credited.

Editor: Hoi Yan Tong

Cite as: Dapia I., Tabakov A., Espinosa Roman L., Díaz L., Arias Lajara P., Melgosa M., Melgar A., Fernandez C., Herranz Estellés A., Lapunzina P., Borobia AM., Carcas AJ., Effect of the CYP3A5, CYP3A4, CYP3A7, ABCB1, POR and NR1I2 genes in the pharmacokinetics of tacrolimus in a pediatric cohort with stable serum concentrations after renal transplantation: study protocol. IBJ Clin Pharmacol 2017 1(1):e0005.

Funding: The authors have no financial relationships relevant to this article to disclose.

Competing Interests: The authors have no financial relationships relevant to this article to disclose 


\section{Background}

Therapeutic response to pharmacological therapy in humans shows large intra and interindividual variability both in treatment efficacy and adverse drug reactions (ADR). Part of this variability can be explained by clinical features such as the stage of the disease or concomitant medications as well as patient age, sex, weight, liver and kidney function among others. However, it has been reported that interindividual variability in drug bioavailability and metabolism as well as differences in molecular and cellular drug targets (both controlled by genetic mechanisms) may have an even more important specific weight over therapeutic response to pharmacological treatments ${ }^{(1)(2)}$

The fast development of molecular techniques and bioinformatics has allowed the identification and characterization of many of the genetic factors explaining the interindividual variability in drug response. The increasing evidence supporting the association of genetic variation to drug efficacy and toxicity heterogeneity has led to the implementation of a Pharmacogenetic (PhGx) approach in the clinical setting which aims to achieve the individualization of therapies by the identification of the most suitable drug and dose for each patient.

Imunosuppressive agents prescribed for renal transplant recipients show a high interindividual pharmacokinetic variability making the individualization of the therapeutic plan necessary to optimize the treatment and avoid graft loss and toxicity. Therapeutic Drug Monitoring (TDM) is mandatory in those drugs showing a narrow therapeutic range such as the calcineurin inhibitor (CNI) tacrolimus (FK506, TAC) ${ }^{(3)}$. Specifically, in pediatric population interindividual variability in TAC metabolism is significantly affected by the gradual maturation of enzyme systems. These differences in metabolic capacity as well as other physiological differences between children and adults lead to higher dose requirements $(\mathrm{mg} / \mathrm{kg})$ in children for achieving similar plasmatic concentrations $^{(4)}$.

TAC is a substrate of the drug-efflux pump ABCB1 or P-glycoprotein (encoded by the $A B C B 1$ gene), which is expressed at high concentrations in the small intestine and thought to be related to TAC heterogeneous bioavailability. Following absorption, TAC is metabolized to up to 15 active and inactive metabolites in the liver, intestine and to a limited degree in the kidney by CYP3A isozymes (predominantly CYP3A5 with CYP3A4 contribution). Interindividual differences in CYP3A activity are the most important determinants of the variability in TAC clearance. However, genetic polymorphisms in other enzymes responsible of the regulation and activation of these drug-metabolizing enzymes such as the P450 oxidoreductase (POR) or the
Nuclear Receptor Subfamily 1 Group I Member 2 (NR1I2) may explain residual variability in the response to $\mathrm{TAC}^{(5)}$.

The human CYP3A subfamily (CYP3A4, CYP3A5, CYP3A7 and CYP43) plays a dominant role in the metabolic elimination of drugs, other xenobiotic compounds and endogenous molecules from the body. These enzymes are encoded by the CYP3A4, CYP $3 A 5$ and CYP3A7 genes, located on chromosome 7. CYP3A4 is the dominant CYP3A enzyme in the adult liver and small intestine. CYP3A5 is also found in the adult liver but with a more variable pattern of expression. In addition, CYP3A5 is also polymorphically expressed in the fetal liver. CYP3A7 is the major fetal liver CYP3A enzyme and its expression generally decreases after birth giving rise to its adult form, CYP3A4. However, CYP3A7 has also been detected in some adults and it may contribute to drug/xenobiotic clearance ${ }^{(6)}$.

\section{CYP3A5 gene}

Genetic polymorphisms in the CYP3A5 gene explain $40-50 \%$ of the variability in TAC dose requirements ${ }^{(3)}$. It has been reported that the strongest predictor of TAC dosing requirements is the rs776746 SNP (c.219-237A $>$ G; CYP3A5*3 allele; $M A F=0,38 \mathrm{~T}$ ) in intron 3. This variant creates an alternative splice site in the pre-mRNA and therefore production of aberrant mRNA resulting in truncation of the translated protein and a severe decrease of functional CYP3A5 enzyme. Homozygous carriers of the $G$ allele of this SNP $(\mathrm{CYP} 3 \mathrm{~A} 5 * 3 / * 3)$ are referred to as CYP3A5 non-expressers in contrast to $\mathrm{CYP} 3 \mathrm{~A} 5 * 1$ carriers (known as CYP3A5 expressers) who show normal protein function and therefore lower trough concentrations related to higher metabolic rates ${ }^{(7-9)}$. This association between CYP3A5 genotype and TAC metabolism has been replicated many times in a variety of different studies. However, the contribution of this genetic variant to TAC pharmacokinetic variability specifically in paediatric population has not been widely reported yet. Turolo et al. (2010) performed a study in teenagers undergoing kidney transplantation and found that carriers of the CYP3A $5 * 1$ allele (CYP3A5 expressers) required a TAC loading dose that was 1,5 times higher than CYP3A5 non-expressers ${ }^{(10)}$. The $* 1$ allele has also been related to a slower achievement of therapeutic levels. Similar results were obtained by Durand et al. in a paediatric cohort $^{(11)}$.On the other hand, Shillbayeh et al. (2013) found this association only during the first 3 months after transplantation, however this may be due to CYP3A5 induction because of corticosteroid comedications or the low frequency of the $* 1$ allele in their cohort (12). As TAC is metabolized by both intestinal and hepatic CYP3A5 enzymes, the combined contribution of CYP3A5 expression in the native intestine and liver 
allograft is likely to influence the pharmacokinetics of TAC in liver transplant. However, due to the short number of studies, up to date results have been inconclusi$\mathrm{ve}^{(3)}$.

Other CYP3A5 SNPs have been reported in association to TAC pharmacokinetics, including the rs 10264272 (c.624G $>$ A; CYP3A5*6; MAF=0,04 T) and rs41303343 (c.1035_1036insT; CYP3A5*7; MAF=0,03 A) encoding nonfunctional proteins as well as the rs41279854 (c. $1337 \mathrm{~T}>\mathrm{C}$; CYP3A5*3K; MAF $<0,01 \mathrm{G}$ ) encoding a protein with decreased activity. The rs55965422 (c. $432+2 \mathrm{~T}>\mathrm{C} ; \mathrm{CYP} 3 \mathrm{~A} 5 * 5 ; \mathrm{MAF}<0,01 \mathrm{G})$ has also been reported affecting the intron 5 splicing donor site of the gene, however the in vitro results revealing the enzymatic activity of the encoded protein were unclear ${ }^{(13-15)}$.

\section{CYP3A4 gene}

The CYP3A4 SNPs rs2740574 (c.-392G $>$ A; CYP3A4*1B; $\mathrm{MAF}=0,23 \quad$ C) and rs35599367 (c.522-191C $>$ T; CYP3A4*22; MAF=0,01 A) have both been related to TAC pharmacokinetic variability. The rs 2740574 is a promoter variant known to increase gene transcription. Therefore carriers of the CYP3A4*1B allele have shown 35\% lower TAC dose-adjusted through concentrations compared to those homozygous for the $* 1$ allele. However the rs 2740574 was shown to be in linkage disequilibrium (LD) with CYP $3 A 5$ rs776746 and therefore its effect in TAC pharmacokinetics is likely mediated by this $\mathrm{SNP}^{(3)}$. The rs35599367 is located in intron 6 of the CYP3A4 gene and has been related to reduced CYP3A4 enzymatic activity. In fact, a study in paediatric heart transplant patients reported that CYP3A4*22 carriers need $30 \%$ less TAC to reach similar target concentrations compared to CYP3A4 $41 / * 1^{(16)}$. This SNP is not in LD with rs776746 in CYP3A5 and therefore its contribution to TAC metabolism is independent from the $\mathrm{CYP} 3 \mathrm{~A} 5 * 3$ allele. A combination of the CYP3A4 and CYP3A5 genotypes has been proposed in order to predict TAC dosing requirements giving rise to three groups: Ultrarapid (CYP3A5 expressers and CYP3A4*1/*1), intermediate (CYP3A5 non-expressers and CYP3A $4 * 1 / * 1)$ and poor (CYP3A5 non-expressers and CYP3A4*22 carriers) CYP3A metabolizers (17-19). The rs28371759 (c.875T $>$ C; CYP3A4*18; MAF $<0,01$ $\mathrm{G})$ in intron 10 of the $C Y P 3 A 4$ gene has also been related to TAC pharmacokinetics ${ }^{(3)}$. Despite not having evidence of a direct association between the rs4646438 (c.827_828insA; CYP3A4*6) and rs55785340 (c.664T $>$ C; CYP3A4*2; MAF $<0,01$ G) TAC pharmacokinetics, Hsieh et al. (2001) reported that these SNPs were associated with reduced CYP3A4 enzymatic activity (in vivo and in vitro respectively) and may therefore affect TAC concentrations ${ }^{(20)}$.

Recently Werk et al. (2014) described a kidney transplant patient with extremely high TAC plasma trough levels during immunosuppressant therapy and a novel $C Y P 3 A 4$ variant resulting in a premature stop codon in CYP3A4 exon 9, rs138105638 (c.802 C>T; CYP3A4*26) resulting in a complete failure of the CYP3A4 locus. In addition, this patient was a CYP3A5 non-expressor and therefore showed a complete failure of the CYP3A enzyme activity and unusually low TAC dosing requirements ${ }^{(21)}$.

\section{CYP3A7 gene}

CYP3A7 is the most abundant CYP isoform and accounts for up to $50 \%$ of the total P450 in embryonic, fetal and neonate livers. Generally its expression decreases after birth giving rise to its adult form, CYP3A4; however it has been reported that individuals carrying the CYP3A7*1C allele that contains the proximal $\mathrm{PXR} / \mathrm{CAR}$ element of $C Y P 3 A 4$ express significant levels of CYP3A7 in their adult life which could therefore contribute to TAC metabolism ${ }^{(22)}$. 5 SNPs define the CYP2C7*1C allele: rs11568824 (c.-291G>T), rs45494802, rs45575938 (c. $-282 \mathrm{~T}>\mathrm{C} ; \quad \mathrm{MAF}=0,01)$, rs45467892 (c. $-281 \mathrm{~A}>\mathrm{T}$; $\mathrm{MAF}=0,01 \mathrm{~A}), \quad \mathrm{rs} 11568825$ (c. $-270 \mathrm{~T}>\mathrm{G}), \mathrm{rs} 11568826$ (c.-262T $>\mathrm{A}$ ) and $\mathrm{rs} 45446698$ (c. $-232 \mathrm{~A}>\mathrm{C} ; \mathrm{MAF}=0,02 \mathrm{G})$. This allele was reported at a frequency of $3 \%$ in Caucasian populations and $6 \%$ in African Americans ${ }^{(6)}$.

Other SNPs in CYP3A7 5'-flanking region have been reported such as rs45465393 (c.-314C $>\mathrm{T}$; CYP3A7*1B; $M A F<0,01$ A), rs55798860 (c. $-91 G>A$; $\mathrm{CYP} 3 \mathrm{~A} 7 * 1 \mathrm{D} ; \quad \mathrm{MAF}=0,01 \mathrm{~T})$ and $\mathrm{rs} 28451617$ (c.-49G>A ; CYP3A7*1E; MAF=0,04 T). However, these variants occur in regions outside those associated with the regulation of CYP3A transcription (6). Another common CYP3A7 SNP is the rs2257401 (c.1226C $>\mathrm{G}$; $\mathrm{CYP} 3 \mathrm{~A} 7 * 2 ; \mathrm{MAF}=0,33 \mathrm{C}$ ), associated with higher drug metabolic rates but only in fetal livers. Lee et al. (2007) reported a novel $C Y P 3 A 7$ variant in a human fetal liver, (c.141_142insT; CYP3A7*3), that causes a premature termination of $C Y P 3 A 7$ translation which may result in a null phenotype ${ }^{(23)}$.

\section{4. $A B C B 1$ gene}

$\mathrm{ABCB} 1$ has been related to the low bioavailability of TAC as well as to TAC distribution throughout the body and its excretion into bile and urine ${ }^{(5)}$. However, associations between TAC pharmacokinetics and variations in $A B C B 1$ are controversial. The rs2032582 (c. $2677 \mathrm{~T}>\mathrm{G} / \mathrm{A} ; \mathrm{ABCB} 1 * 2 ; \mathrm{MAF}=0,05 \mathrm{~T}), \quad \mathrm{rs} 1045642$ (c. $3435 \mathrm{~T}>\mathrm{C} ; \mathrm{ABCB} 1 * 2 ; \mathrm{MAF}=0,40 \mathrm{~A})$ and $\mathrm{rs} 1128503$ (c. $1236 \mathrm{~T}>\mathrm{C} ; \mathrm{ABCB} 1 * 2 ; \mathrm{MAF}=0,42 \mathrm{~A}$ ) which are likely in LD are the most studied SNPs, however results are conflicting and there is no consistent evidence for an association between TAC adjusted-through concentrations and these variants ${ }^{(24,25)}$. Some studies report a sig- 
nificant association between $\mathrm{rs} 1045642$ and TAC dose $^{(8,}$ 26), however this association was not confirmed by other authors $^{(27)}$.

$\mathrm{ABCB} 1$ is also expressed in the membrane of lymphocytes so it may play a role in affecting the intracellular concentrations of TAC within this cells. Indeed, Capron et al (2010) reported an association between this SNPs and intracellular PBMC TAC concentrations (probably due to a reduction in P-glycoprotein expression and function). In addition, this association was not correlated with TAC blood concentrations, demonstrating that blood concentration may not accurately reflect the level of TAC available for immunosuppression ${ }^{(28)}$. $A B C B 1$ SNPs have also been related to TAC nephrotoxicity as P-gp expression in renal cells could determine the amount of TAC entering these cells and therefore tissue concentrations $^{(29)}$. However, this association was not replicated by other studies ${ }^{(30)}$.

Kroetz et al. described the $* 13$ haplotype ${ }^{(31)}$. $\mathrm{ABCB} 1 * 13$ is indistinguishable from the *2 haplotype in terms of coding region and aminoacid sequence but different in 3 intronic variants: rs10276036 (c.1000-44G $>$ A; $\mathrm{ABCB} 1 * 13 ; \mathrm{MAF}=0,43 \mathrm{~A}), \mathrm{rs} 2235033$ (c. $1554+24 \mathrm{~T}>\mathrm{C}$; $\mathrm{ABCB} 1 * 13$; $\mathrm{MAF}=0,47 \quad \mathrm{G})$ and $\mathrm{rs} 2235013$ (c. $1725+38 \mathrm{G}>\mathrm{A}$; $\mathrm{ABCB} 1 * 13 ; \mathrm{MAF}=0,47 \mathrm{~T}$ ). This haplotype does not affect $\mathrm{ABCB} 1$ protein function.

\section{POR gene}

P-450 oxidoreductase (POR) enables the activity of CYP3A enzymes and it is essential for CYP-mediated drug oxidation. The rs1057868 (c.1508C $>$ T; POR*28; $\mathrm{MAF}=0,29 \mathrm{~T}$ ) affects the electron binding domain of POR and therefore modifies POR modulation of the CYP3A emzymes. Indeed, it has been reported that homozygus carriers of the POR*28 allele show an increased CYP3A in vivo activity ${ }^{(32)}$. In pediatric population, a recent study in 43 transplant children showed that heterozygous and homozygous carriers of the *28 allele show TAC concentration/dose ratios 20,2\% lower than POR* $1 / * 1$ individuals. An association between POR rs2868177 (c. $188+6405 \mathrm{~A}>\mathrm{G} ; \mathrm{MAF}=0,40 \mathrm{G}$ ) and $\mathrm{CYP}$ activity has also been described as it strongly related to warfarin maintenance dose variations ${ }^{(33)}$.

\section{NR1I2 gene}

The Nuclear Receptor Subfamily 1 Group I Member 2 (NR1I2) encodes the human pregnane $\mathrm{X}$ receptor (PXR), responsible of the upstream regulation of the CYP3A enzymes. The promoter NR1I2 SNP rs3814055 (c. $-1570 \mathrm{C}>\mathrm{T} ; \mathrm{MAF}=0,32 \mathrm{~T}$ ) has been related to a signi ficantly increased exposure to TAC. It has been suggested that this variant produces a downregulation of PXR and therefore decreased CYP metabolic activity resulting in decreased clearance of TAC ${ }^{(34)}$.

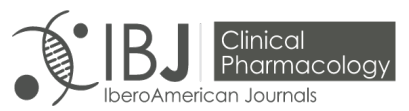

In conclusion, TAC efficacy and toxicity show a great variability both in adult and pediatric population. Part of this variability can be explained by genetic variation in the CYP3A5, CYP $3 A 4$ and CYP3A7, POR, NR1I2 and $A B C B 1$ genes. Therefore our group proposes this study for the evaluation of SNP affecting these genes could help us in the design and optimization of new strategies, such as dosing algorithms for treatment individualization and optimization (FC/HULP_002/2014).

\section{Methods}

\section{Design and setting}

This study was designed as a unicenter retrospective cross-sectional study to evaluate the contribution of genetic polymorphisms in TAC metabolism related genes in the pharmacokinetics of TAC in pediatric patients with stable serum concentrations after renal transplantation. The study will be performed in La Paz University Hospital in Madrid (Spain). This study has an estimated duration of 24 months. A Flowchart of the study procedures can be found in Figure 1.

\section{Study population}

The target population is a cohort of pediatric patients after renal transplantation referred to the Pediatric Nephrology Division of La Paz University Hospital and following a pharmacological treatment with TAC. Peripheral blood samples will be drawn for molecular analysis and AUC estimation.

\section{Inclusion criteria}

- Male and female patients who underwent a renal transplantation procedure.

- Under 18 years of age.

- Follow stable immunosuppressive treatment with TAC. Treatment is considered stable when variations in the 3 last blood concentrations determinations are $<30 \%$, being these determinations separated by 1 week.

- Patients give written consent to participate in the study once they have received information on the design, goals and possible risk of the study and they are made aware that they can withdraw from the study at any time.

\section{Exclusion criteria}

- Inability to understand directions or lack of collaboration during the study.

\section{Objectives}

\section{a) Primary}




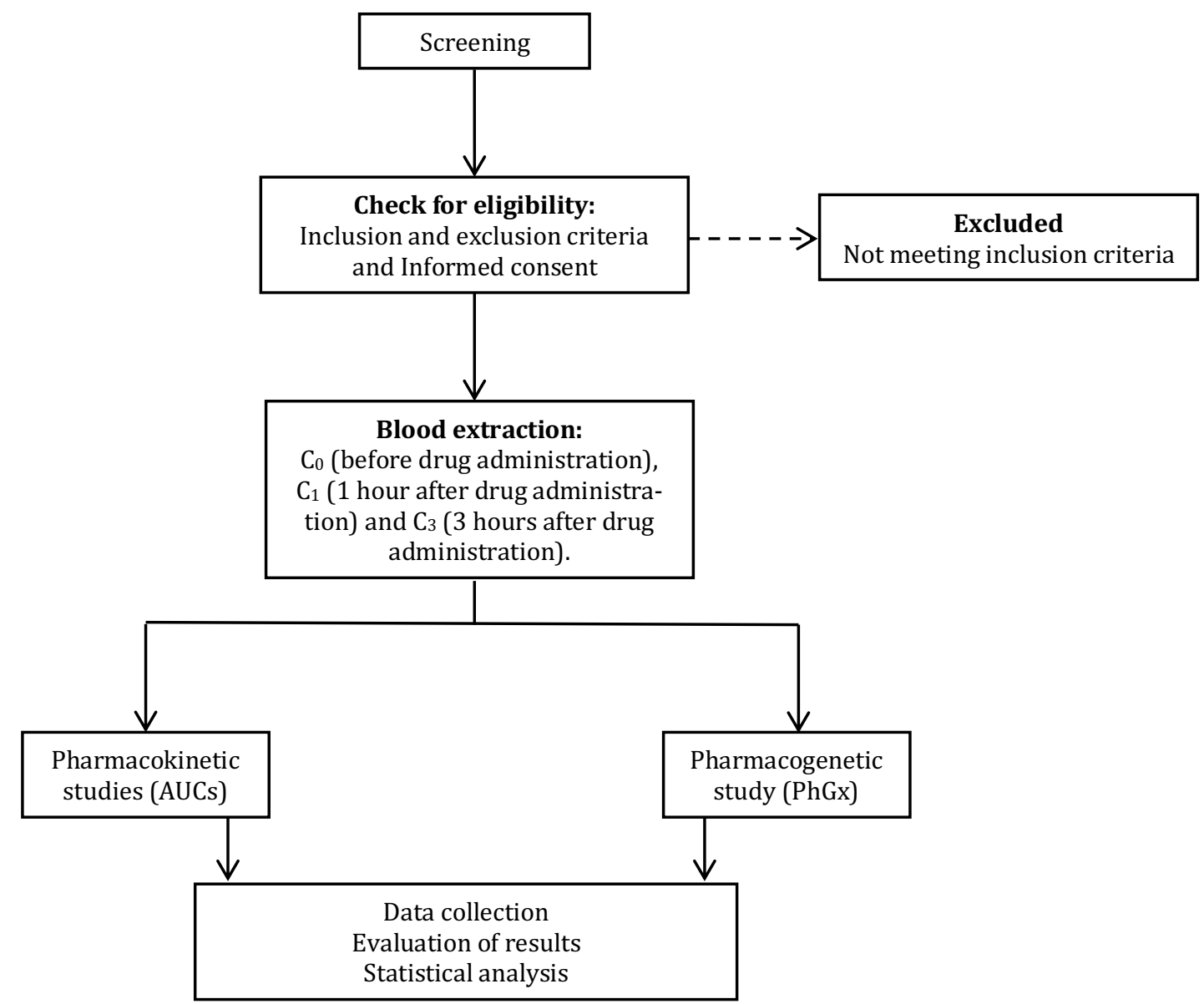

- To evaluate the contribution of genetic variation in the $C Y P 3 A 4, C Y P 3 A 5, C Y P 3 A 7, P O R$, $N R 112$ and $A B C B 1$ genes to TAC pharmacokinetics.

\section{b) Secondary}

- To perform molecular analysis and characterization of $C Y P 3 A, A B C B 1, P O R$ and NR1I2 haplotypes in a pediatric cohort after renal transplantation.

- To evaluate the contribution of genetic variation (in addition to other individual features) to TAC heterogeneity in clinical response and related adverse reactions by mathematical modelling.

\section{Study timeline}

The study will be distributed as shown in Table 1 .

\section{a) Inclusion phase}

- Recruitment of a cohort of pediatric patients referred to the Pediatric Nephrology Division after renal transplant. Patients will be checked to ensure they meet the selection criteria.
- $\quad$ Before any procedure is performed patients and relatives will be informed (verbally and in written by the patient information sheet). Informed consent sheets will be gathered. An example of the Informed Consent sheets can be found in the Supplementary Material.

- Once patients and relatives have consent to participation in the study clinical and demographic information will be collected by personal interviews and review of medical history.

- 3 blood samples will be taken from each patient for a TAC AUC study: C0, C1 (1 hour) and C3 (3 hours).

- In order to minimize the number of blood extractions, one of the previous samples will be used for the PhGx studies.

\section{b) Result collection and analysis}

- PhGx tests will be performed. Results will be collected in electronic databases for subsequent statistical analysis.

\section{c) Results dissemination}

- Preparation of a final report. 

Table 1 stable serum foncentrations after renal transplantation: study protocol.

April 2014-January 2017 January-March 2017 April 2017

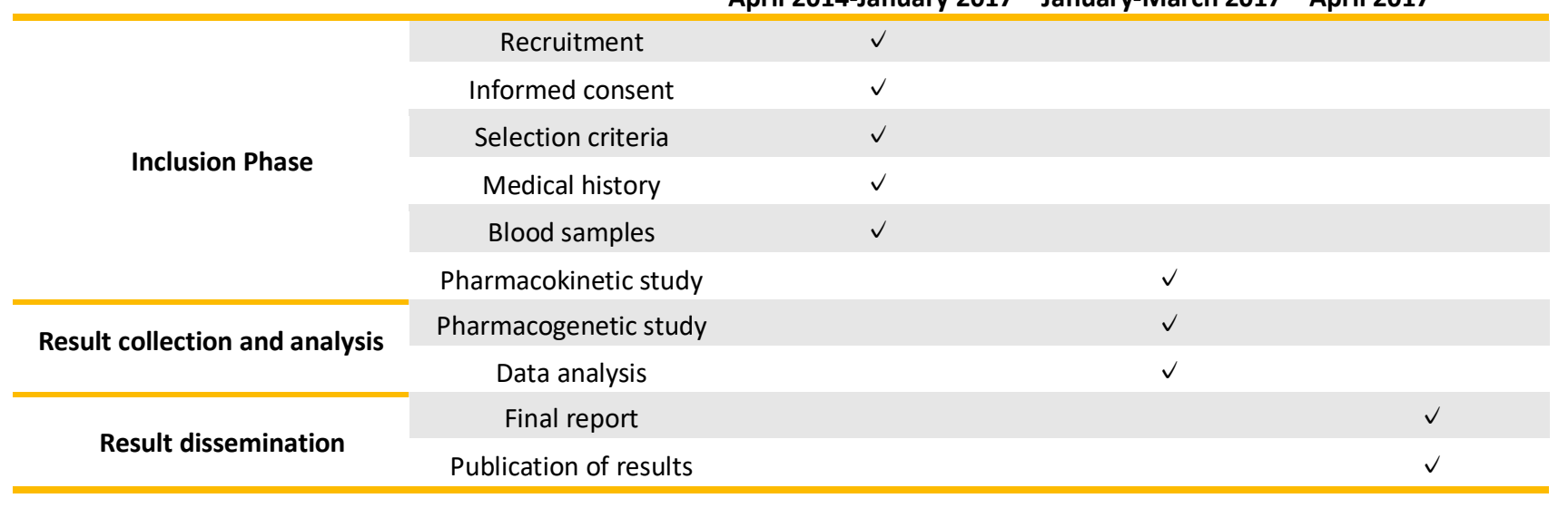

- Publication of the results in scientific journals.

- Communications in scientific meetings and conferences.

\section{Data collection}

Patient's clinical information will be collected by medical history review and a personal interview with patients and relatives. Three blood samples will be drawn for AUC and PhGx studies. Information will be recorded in the Case Report Form (CRF).

\section{a) Pharmacogenetics studies}

One of the blood samples obtained for the pharmacokinetic study will be used for DNA extraction and genotyping of various SNP in the following genes related to TAC metabolism and pharmacokinetics: CYP3A4, CYP3A5, CYP3A7, POR, NR1I2 and $A B C B 1$ in the Medical and Molecular Genetics Institute (INGEMM) in La Paz University Hospital. Table 2 shows the final selection of SNPs for TAC PhGx analysis. After the assessment of different molecular techniques we finally selected the OpenArray ${ }^{\circledR}$ technique with a custom format: PharmArray ${ }^{\circledR}$ as well as direct sequencing for those SNPs which were not included in the design.

\section{b) Pharmacokinetic study}

Three blood samples will be collected from each patient: One before drug administration $(\mathrm{C} 0)$, and two after 1 and 3 hours respectively (C1 and $\mathrm{C} 3)$ in order to calculate TAC abbreviated AUC as an indicator of total drug exposure. This method is completely validated and is performed on a daily basis in many patients (35). These determinations will be performed in the Clinical Pharmacology Department of La Paz University Hospital by an automatized immunoassay method (Architect ${ }^{\circledR}$ ), which is generally used for TDM of immunosuppressive drugs.

\section{Case Report Form (CRF)}

Each patient's CRF will include the following information:

Age, sex, intercurrent diseases that may modify the activity of TAC metabolizing enzymes as well as possible concomitant treatments with enzyme inducers or inhibitors (erythromycin, phenobarbital, itraconazole, diltiazem, Ritonavir/Lopinavir, etc). TAC concentrations will also be recorded (abbreviated AUC in $\mathrm{C} 0, \mathrm{C} 1$ and $\mathrm{C} 3$ ). In the event of TAC-related adverse reactions, an independent evaluation of immunosuppressive treatment will be performed.

\section{Withdrawal criteria}

Patients can discontinue their participation in the study at any time. The research doctor, in his or her opinion or judgment, may also withdraw a patient from the study if required by the patient's clinical situation or if the patient does not comply with the protocol. The motive and justification of the withdrawal will be reflected in the study development.

\section{Sample size calculation}

Due to the exploratory nature of this study our aim is to include all pediatric patients referred to the Pediatric Nephrology Division of La Paz University Hospital. Taking into account $\sim 20 \%$ of patients that refuse to collaborate in the study and/or withdrawals, we estimate a number of patients of around 50, this sample size seemed adequate as compared to other previous similar stu$\operatorname{dies}^{(8,12,16)}$.

\section{Data analysis}

Data will be collected in electronic databases for subsequent analysis:

a. We will perform a descriptive analysis of socio-demographic factors, personal records and 
Table 2. Final selection of SNPs included in the TAC PhGx study. Information about SNPs coordinates and Minor allele Frequencies (MAF) is shown.

\begin{tabular}{|c|c|c|c|c|}
\hline Gene & SNPs (\#rs) & cDNA & Genomic Location & MAF \\
\hline \multirow{6}{*}{$A B C B 1$} & rs2032582 & c. $2677 \mathrm{~T}>\mathrm{G} / \mathrm{A}$ & Chr7:87531302 & $0,05 \mathrm{~T}$ \\
\hline & rs1045642 & c. $3435 \mathrm{~T}>\mathrm{C}$ & Chr7:87509329 & $0,40 \mathrm{~A}$ \\
\hline & rs1128503 & c. $1236 \mathrm{~T}>\mathrm{C}$ & Chr7:87550285 & $0,42 \mathrm{~A}$ \\
\hline & rs10276036 & c. $1000-44 G>A$ & Chr7:87550882 & $0,43 \mathrm{~A}$ \\
\hline & rs2235033 & c. $1554+24 \mathrm{~T}>\mathrm{C}$ & Chr7:87549827 & $0,47 \mathrm{G}$ \\
\hline & rs2235013 & c. $1725+38 G>A$ & Chr7:87549310 & $0,47 \mathrm{~T}$ \\
\hline \multirow{6}{*}{ CYP3A4 } & rs55785340 & c. $664 \mathrm{~T}>\mathrm{C}$ & Chr7:99768360 & $<0,01 \mathrm{G}$ \\
\hline & rs4646438 & c.827_828insA & Chr7:99766411_99766412 & - \\
\hline & rs 2740574 & c. $-392 \mathrm{G}>\mathrm{A}$ & Chr7:99784473 & $0,23 \mathrm{C}$ \\
\hline & rs35599367 & c.522-191C>T & Chr7:99768693 & $0,01 \mathrm{~A}$ \\
\hline & rs28371759 & c. $875 \mathrm{~T}>\mathrm{C}$ & Chr7:99764003 & $<0,01 \mathrm{G}$ \\
\hline & rs138105638 & c. $802 \mathrm{C}>\mathrm{T}$ & Chr7:99766440 & - \\
\hline \multirow{5}{*}{ CYP3A5 } & rs776746 & c. $219-237 A>G$ & Chr7:99672916 & $0,38 \mathrm{~T}$ \\
\hline & rs55965422 & c. $432+2 T>C$ & Chr7:99666950 & $<0,01 \mathrm{G}$ \\
\hline & rs10264272 & c. $624 \mathrm{G}>\mathrm{A}$ & Chr7:99665212 & $0,04 \mathrm{~T}$ \\
\hline & rs41303343 & c.1035_1036insT & Chr7:99652770_99652771 & $0,03 \mathrm{~A}$ \\
\hline & rs41279854 & c. $1337 \mathrm{~T}>\mathrm{C}$ & Chr7:99650149 & $0,01 \mathrm{G}$ \\
\hline \multirow{12}{*}{ CYP3A7 } & rs45465393 & c. $-314 C>T$ & Chr7:99735407 & $<0,01 \mathrm{~A}$ \\
\hline & rs11568824 & c. $-291 \mathrm{G}>\mathrm{T}$ & Chr 7:99735384 & - \\
\hline & rs45494802 & c. $-284 \mathrm{~T}>\mathrm{A}$ & Chr7:99735377 & $0,01 \mathrm{~T}$ \\
\hline & rs45575938 & c. $-282 \mathrm{~T}>\mathrm{C}$ & Chr7:99735375 & $0,01 \mathrm{G}$ \\
\hline & rs45467892 & c. $-281 \mathrm{~A}>\mathrm{T}$ & Chr 7:99735374 & $0,01 \mathrm{~A}$ \\
\hline & rs11568825 & c. $-270 T>G$ & Chr7:99735363 & - \\
\hline & rs11568826 & c. $-262 T>A$ & Chr 7:99735355 & - \\
\hline & rs45446698 & c. $-232 A>C$ & Chr7:99735325 & $0,02 \mathrm{G}$ \\
\hline & rs55798860 & c. $-91 \mathrm{G}>\mathrm{A}$ & Chr7:99735184 & $0,01 \mathrm{~T}$ \\
\hline & rs28451617 & c. $-49 \mathrm{G}>\mathrm{A}$ & Chr7:99735142 & $0,04 \mathrm{~T}$ \\
\hline & rs2257401 & c. $1226 C>G$ & Chr7:99709062 & $0,33 \mathrm{C}$ \\
\hline & - & c.141_142insT & Chr7:99731082_99731083 & - \\
\hline \multirow{2}{*}{ POR } & rs1057868 & c. $1508 \mathrm{C}>\mathrm{T}$ & Chr7:75985688 & $0,29 \mathrm{~T}$ \\
\hline & rs 2868177 & c. $188+6405 A>G$ & Chr7:75960585 & $0,40 \mathrm{G}$ \\
\hline NR1/2 & rs3814055 & c. $-1570 C>T$ & Chr3:119781188 & $0,32 \mathrm{~T}$ \\
\hline
\end{tabular}

Transcripts IDs: ABCB1 (NM_000927), CYP3A4 (NM_001202855), CYP3A5 (NM_000777), CYP3A7 (NM_000765), POR (NM_000941) and NR1/2 (NM_022002). Genome version: Human GRCh38.p7. 
concomitant drugs, transplant and immunosuppression information, pharmacokinetic parametres (abbreviated AUC) and PhGx data (allelic and genotypic frequencies). Data will be expressed as incidence (number/percentage) or average (with standard deviation, SD), as applicable.

b. A univariate analysis will be performed for the evaluation of the relationship between kinetic parameters (drug exposure-AUC) and the other independent variables (including $\mathrm{PhGx}$ ). T-Student, ANOVA and bivariate correlation (or its corresponding non-parametric test) will be applied. For the evaluation of each separate variable in AUC a univariate lineal regression will be performed and R2 and AIC will be used for comparison of the different variables.

c. A multivariate regression analysis will be used for building an explicative model of the patient's clinical response, being the independent variables the selected genetic variants and clinical a demographic features of the patients and the dependent variable the pharmacokinetic results (AUC).

\section{Ethical aspects}

Researchers will abide strictly to the provisions of this protocol as well as to the Good Clinical Practice guidelines. This project will be carried according to the recommendations for clinical studies and drug evaluation in humans as reflected in the Declaration of Helsinki (reviewed in successive world meetings) and in the current Spanish legislation on clinical studies (RD 223/2004) and patient data confidentiality. This study has been approved by the Clinical Research Ethics Committee of the La Paz University Hospital (Madrid, Spain).

Patients will receive verbal and written information about the study and the possible associated risks before being included in the study and a written consent form will be collected. Included patients will be underage and therefore different information sheets and informed consent forms suitable for the different age group will be designed. Patients will be able to withdraw from this study at any moment. Results obtained in this study are considered confidential and will be treated strictly as such.

\section{Discussion}

Tacrolimus is the most commonly used calcineurin inhibitor (with more than $90 \%$ of kidney transplants performed with TAC as immunosuppressive agent $)^{(36)}$. However, TAC therapy is complicated by its narrow therapeutic window requiring TDM to optimize treatment and avoid graft loss and toxicities ${ }^{(37-39)}$. Despite TDM, a high inter and intraindividual pharmacokinetic variability has been reported in transplant patients treated with TAC. This pharmacokinetic variability depends on many clinical co-variants such as age, sex, weight, serum levels of albumin, haematocrit and haemoglobin, concomitant medications or post-operative time. However, it has been reported that genetic variations in the genes encoding proteins related to TAC metabolism and disposition may contribute in a high degree to this heterogeneity of responses ${ }^{(40-42)}$.

Recently it has been proposed the implementation of dosing algorithms in the clinical routine that include all of these variables for increasing both the efficacy and safety of TAC in renal transplant patients. Several dosing algorithms have been proposed ${ }^{(43-45)}$. However, these algorithms do not incorporate the complete TAC related $\mathrm{PhGx}$ profile and therefore are unable to accurately determine TAC clearance.

Our project aims to evaluate the individual contribution of the most common and important genetic variants reported in association to TAC pharmacokinetics in order to identify new variables that could be incorporated in dosing algorithms and therefore improve TAC dosing and security.

Passey et al. included $C Y P 3 A 5$ genotype as a variable in their TAC dosing algorithm. Genetic polymorphisms in the $C Y P 3 A 5$ gene explain $40-50 \%$ of the variability in TAC dose requirements. However TAC presents an extensive hepatic metabolism by other cytochrome $\mathrm{P} 450$ enzymes (such as CYP3A4 and CYP3A7), and its bioavailability is also influenced by the multidrug resistance genotypes $(A B C B 1)^{(8,46)}$. In addition, recent studies have related variants in POR and NRII2 with TAC pharmacokinetics, as these genes encode proteins related to the regulation of the CYP3A enzymes ${ }^{(3)}$. CYP3A7 is the major fetal liver metabolizing enzyme. Its expression generally decreases after birth, however it has been reported that individuals carrying the CYP3A $7 * 1 \mathrm{C}$ allele express significant levels of CYP3A7 in their adult life. We therefore included $C Y P 3 A 7$ screening in our study as we hypothesize that this protein, especially in pediatric population, may contribute in some way to TAC metabolism $^{(22)}$.

In summary, the proposed study aims to review and identify the specific contribution of genetic variation in these genes in relation to TAC pharmacokinetics in pediatric population. Preemptive screening of these genes may result an informative tool that could help clarifying TAC heterogeneity of responses and help in the prevention of toxicities and graft loss as well as in the optimization of TAC dose and efficiency.

\section{Acknowledgement}

This work has had the support of the Spanish Clinical Research Network (SCReN), funded by IS-CIII-Subdirección General de Evaluación y Fomento de la Investigación (project PT13/0002/0015), integrated in Plan Estatal de I+D+I 2013-2016, and 
co-financed by Fondo Europeo de Desarrollo Regional (FEDER).

\section{References}

[1] McLeod HL. Pharmacogenetics: more than skin deep. Nature genetics. 2001;29(3):247-8.

[2] Evans WE, Relling MV. Pharmacogenomics: translating functional genomics into rational therapeutics. Science. 1999;286(5439):487-91.

[3] Barbarino JM, Staatz CE, Venkataramanan R, Klein TE, Altman RB. PharmGKB summary: cyclosporine and tacrolimus pathways. Pharmacogenetics and genomics. 2013;23(10):563-85.

[4] Martial LC, Verstegen RH, Cornelissen EA, Aarnoutse RE, Schreuder MF, Bruggemann RJ. A preliminary study searching for the right dose of tacrolimus in very young $(</=4$ years $)$ renal transplant patients. J Pharm Pharmacol. 2016;68(11):1366-72.

[5] Tang JT, Andrews LM, van Gelder T, Shi YY, van Schaik RH, Wang LL, et al. Pharmacogenetic aspects of the use of tacrolimus in renal transplantation: recent developments and ethnic considerations. Expert Opin Drug Metab Toxicol. 2016;12(5):555-65.

[6] Lamba JK, Lin YS, Schuetz EG, Thummel KE. Genetic contribution to variable human CYP3A-mediated metabolism. Adv Drug Deliv Rev. 2002;54(10):1271-94.

[7] Mourad M, Mourad G, Wallemacq P, Garrigue V, Van Bellingen C, Van Kerckhove V, et al. Sirolimus and tacrolimus trough concentrations and dose requirements after kidney transplantation in relation to CYP3A5 and MDR1 polymorphisms and steroids. Transplantation. 2005;80(7):977-84.

[8] Roy JN, Barama A, Poirier C, Vinet B, Roger M. Cyp3A4, Cyp3A5, and MDR-1 genetic influences on tacrolimus pharmacokinetics in renal transplant recipients. Pharmacogenetics and genomics. 2006;16(9):659-65.

[9] Thervet E, Anglicheau D, King B, Schlageter MH, Cassinat B, Beaune P, et al. Impact of cytochrome p450 3A5 genetic polymorphism on tacrolimus doses and concentration-to-dose ratio in renal transplant recipients. Transplantation. 2003;76(8):1233-5.

[10] Turolo S, Tirelli AS, Ferraresso M, Ghio L, Belingheri M, Groppali E, et al. Frequencies and roles of CYP3A5, CYP3A4 and ABCB1 single nucleotide polymorphisms in Italian teenagers after kidney transplantation. Pharmacol Rep. 2010;62(6):1159-69.

[11] Durand P, Debray D, Kolaci M, Bouligand J, Furlan V, Fabre M, et al. Tacrolimus dose requirement in pediatric liver transplantation: influence of CYP3A5 gene polymorphism. Pharmacogenomics. 2013;14(9):1017-25.
[12] Shilbayeh S, Zmeili R, Almardini RI. The impact of CYP3A5 and MDR1 polymorphisms on tacrolimus dosage requirements and trough concentrations in pediatric renal transplant recipients. Saudi J Kidney Dis Transpl. 2013;24(6):1125-36.

[13] Chou FC, Tzeng SJ, Huang JD. Genetic polymorphism of cytochrome P450 3A5 in Chinese. Drug metabolism and disposition: the biological fate of chemicals. 2001;29(9):1205-9.

[14] Kuehl P, Zhang J, Lin Y, Lamba J, Assem M, Schuetz J, et al. Sequence diversity in CYP3A promoters and characterization of the genetic basis of polymorphic CYP3A5 expression. Nature genetics. 2001;27(4):383-91.

[15] Lee SJ, Usmani KA, Chanas B, Ghanayem B, Xi T, Hodgson E, et al. Genetic findings and functional studies of human CYP3A5 single nucleotide polymorphisms in different ethnic groups. Pharmacogenetics. 2003;13(8):461-72.

[16] Gijsen VM, van Schaik RH, Elens L, Soldin OP, Soldin SJ, Koren G, et al. CYP3A4*22 and CYP3A combined genotypes both correlate with tacrolimus disposition in pediatric heart transplant recipients. Pharmacogenomics. 2013;14(9):1027-36.

[17] Elens L, Bouamar R, Hesselink DA, Haufroid V, van der Heiden IP, van Gelder T, et al. A new functional CYP3A4 intron 6 polymorphism significantly affects tacrolimus pharmacokinetics in kidney transplant recipients. Clinical chemistry. 2011;57(11):1574-83.

[18] Elens L, Capron A, van Schaik RH, De Meyer M, De Pauw L, Eddour DC, et al. Impact of CYP3A4*22 allele on tacrolimus pharmacokinetics in early period after renal transplantation: toward updated genotype-based dosage guidelines. Ther Drug Monit. 2013;35(5):608-16.

[19] Elens L, van Schaik RH, Panin N, de Meyer M, Wallemacq P, Lison D, et al. Effect of a new functional CYP3A4 polymorphism on calcineurin inhibitors' dose requirements and trough blood levels in stable renal transplant patients. Pharmacogenomics. 2011;12(10):1383-96.

[20] Hsieh KP, Lin YY, Cheng CL, Lai ML, Lin MS, Siest JP, et al. Novel mutations of CYP3A4 in Chinese. Drug metabolism and disposition: the biological fate of chemicals. 2001;29(3):268-73.

[21] Werk AN, Lefeldt S, Bruckmueller H, Hemmrich-Stanisak G, Franke A, Roos M, et al. Identification and characterization of a defective CYP3A4 genotype in a kidney transplant patient with severely diminished tacrolimus clearance. Clinical pharmacology and therapeutics. 2014;95(4):416-22.

[22] Sim SC, Edwards RJ, Boobis AR, Ingelman-Sundberg M. CYP3A7 protein expression is high in a fraction of adult human livers and partially associated with the 
CYP3A7*1C allele. Pharmacogenetics and genomics. 2005;15(9):625-31.

[23] Lee SS, Jung HJ, Park JS, Cha IJ, Cho DY, Shin JG. Identification of a null allele of cytochrome P450 3A7: CYP3A7 polymorphism in a Korean population. Mol Biol Rep. 2010;37(1):213-7.

[24] Staatz CE, Tett SE. Clinical pharmacokinetics and pharmacodynamics of tacrolimus in solid organ transplantation. Clin Pharmacokinet. 2004;43(10):623-53.

[25] Tang HL, Xie HG, Yao Y, Hu YF. Lower tacrolimus daily dose requirements and acute rejection rates in the CYP3A5 nonexpressers than expressers. Pharmacogenetics and genomics. 2011;21(11):713-20.

[26] Macphee IA, Fredericks S, Tai T, Syrris P, Carter ND, Johnston A, et al. Tacrolimus pharmacogenetics: polymorphisms associated with expression of cytochrome p4503A5 and P-glycoprotein correlate with dose requirement. Transplantation. 2002;74(11):1486-9.

[27] Tavira B, Gomez J, Diaz-Corte C, Suarez B, Coronel D, Arias M, et al. ABCB1 (MDR-1) pharmacogenetics of tacrolimus in renal transplanted patients: a Next Generation Sequencing approach. Clin Chem Lab Med. 2015;53(10):1515-9.

[28] Capron A, Mourad M, De Meyer M, De Pauw L, Eddour DC, Latinne D, et al. CYP3A5 and ABCB1 polymorphisms influence tacrolimus concentrations in peripheral blood mononuclear cells after renal transplantation. Pharmacogenomics. 2010;11(5):703-14.

[29] Naesens M, Lerut E, de Jonge H, Van Damme B, Vanrenterghem Y, Kuypers DR. Donor age and renal P-glycoprotein expression associate with chronic histological damage in renal allografts. Journal of the American Society of Nephrology : JASN. 2009;20(11):2468-80.

[30] Debette-Gratien M, Woillard JB, Picard N, Sebagh M, Loustaud-Ratti V, Sautereau D, et al. Influence of Donor and Recipient CYP3A4, CYP3A5, and ABCB1 Genotypes on Clinical Outcomes and Nephrotoxicity in Liver Transplant Recipients. Transplantation. 2016;100(10):2129-37.

[31] Kroetz DL, Pauli-Magnus C, Hodges LM, Huang CC, Kawamoto M, Johns SJ, et al. Sequence diversity and haplotype structure in the human ABCB1 (MDR1, multidrug resistance transporter) gene. Pharmacogenetics. 2003;13(8):481-94.

[32] Oneda B, Crettol S, Jaquenoud Sirot E, Bochud M, Ansermot N, Eap CB. The P450 oxidoreductase genotype is associated with CYP3A activity in vivo as measured by the midazolam phenotyping test. Pharmacogenetics and genomics. 2009;19(11):877-83.

[33] Zhang X, Li L, Ding X, Kaminsky LS. Identification of cytochrome $\mathrm{P} 450$ oxidoreductase gene variants that are significantly associated with the interindividual variations in warfarin maintenance dose. Drug Metab Dispos. 2011;39(8):1433-9.

[34] Choi Y, Jiang F, An H, Park HJ, Choi JH, Lee H. A pharmacogenomic study on the pharmacokinetics of tacrolimus in healthy subjects using the DMETTM Plus platform. The pharmacogenomics journal. 2016.

[35] Almeida-Paulo GN, Lubomirov R, Alonso-Sanchez NL, Espinosa-Roman L, Fernandez Camblor C, Diaz C, et al. Limited sampling strategies for tacrolimus exposure (AUC0-24) prediction after Prograf((R)) and Adva$\operatorname{graf}((\mathrm{R}))$ administration in children and adolescents with liver or kidney transplants. Transplant international : official journal of the European Society for Organ Transplantation. 2014;27(9):939-48.

[36] Pulk RA, Schladt DS, Oetting WS, Guan W, Israni AK, Matas AJ, et al. Multigene predictors of tacrolimus exposure in kidney transplant recipients. Pharmacogenomics. 2015;16(8):841-54.

[37] Felipe CR, Silva HT, Machado PG, Garcia R, da Silva Moreira SR, Pestana JO. The impact of ethnic miscegenation on tacrolimus clinical pharmacokinetics and therapeutic drug monitoring. Clin Transplant. 2002;16(4):262-72.

[38] Scott LJ, McKeage K, Keam SJ, Plosker GL. Tacrolimus: a further update of its use in the management of organ transplantation. Drugs. 2003;63(12):1247-97.

[39] Taylor AL, Watson CJ, Bradley JA. Immunosuppressive agents in solid organ transplantation: Mechanisms of action and therapeutic efficacy. Crit Rev Oncol Hematol. 2005;56(1):23-46.

[40] Kuypers DR, Claes K, Evenepoel P, Maes B, Coosemans $\mathrm{W}$, Pirenne J, et al. Time-related clinical determinants of long-term tacrolimus pharmacokinetics in combination therapy with mycophenolic acid and corticosteroids: a prospective study in one hundred de novo renal transplant recipients. Clin Pharmacokinet. 2004;43(11):741-62.

[41] Mourad M, Wallemacq P, De Meyer M, Brandt D, Van Kerkhove V, Malaise J, et al. The influence of genetic polymorphisms of cytochrome $\mathrm{P} 4503 \mathrm{~A} 5$ and $\mathrm{ABCB} 1$ on starting dose- and weight-standardized tacrolimus trough concentrations after kidney transplantation in relation to renal function. Clin Chem Lab Med. 2006;44(10):1192-8.

[42] Undre NA, van Hooff J, Christiaans M, Vanrenterghem Y, Donck J, Heeman U, et al. Low systemic exposure to tacrolimus correlates with acute rejection. Transplant Proc. 1999;31(1-2):296-8.

[43] Passey C, Birnbaum AK, Brundage RC, Oetting WS, Israni $\mathrm{AK}$, Jacobson PA. Dosing equation for tacrolimus using genetic variants and clinical factors. Br J Clin Pharmacol. 2011;72(6):948-57.

[44] Passey C, Birnbaum AK, Brundage RC, Schladt DP, Oetting WS, Leduc RE, et al. Validation of tacrolimus equa- 
tion to predict troughs using genetic and clinical factors. Pharmacogenomics. 2012;13(10):1141-7.

[45] Storset E, Asberg A, Skauby M, Neely M, Bergan S, Bremer S, et al. Improved Tacrolimus Target Concentration Achievement Using Computerized Dosing in Renal Transplant Recipients--A Prospective, Randomized Study. Transplantation. 2015;99(10):2158-66.
[46] Anglicheau D, Verstuyft C, Laurent-Puig P, Becquemont L, Schlageter MH, Cassinat B, et al. Association of the multidrug resistance-1 gene single-nucleotide polymorphisms with the tacrolimus dose requirements in renal transplant recipients. Journal of the American Society of Nephrology : JASN. 2003;14(7):1889-9 